INSTITUTE OF FOOD AND RESOURCE ECONOMICS

UNIVERSITY OF COPENHAGEN

FOI Working Paper

Attending to the reasons for attribute non-attendance in Choice Experiments

Mohammed Hussen Alemu Morten Raun Mørkbak Søren Bøye Olsen Carsten Lynge Jensen 


\section{FOI Working Paper 2011 / 8}

Attending to the reasons for attribute non-attendance in Choice Experiments

Authors: Mohammed Hussen Alemu, Morten Raun Mørkbak, Søren Bøye Olsen, Carsten Lynge Jensen

Institute of Food and Resource Economics

University of Copenhagen

Rolighedsvej 25

DK 1958 Frederiksberg DENMARK

www.foi.life.ku.dk 


\title{
Attending to the reasons for attribute non-attendance in Choice Experiments
}

\author{
Mohammed Hussen Alemu, \\ Morten Raun Mørkbak, \\ Søren Bøye Olsen, \\ Carsten Lynge Jensen \\ University of Copenhagen
}

\begin{abstract}
This paper focuses on behavioural reasons underlying stated attribute nonattendance. In order to identify and incorporate procedures for dealing with heterogeneous attribute processing strategies, we ask respondents follow-up questions regarding their reasons for ignoring attributes. Based on these statements, we conclude that the standard way of assigning a zero impact of ignored attributes on the likelihood is inappropriate. We find that some respondents act in accordance with the passive bounded rationality assumption since they ignore an attribute simply because it does not affect their utility. Excluding these genuine zero preferences, as the standard approach essentially does, might bias results. Other respondents claim to have ignored attributes to simplify choices. However, we find that these respondents have actually not completely ignored attributes. We argue along the rationally adaptive behavioural model that preferences are indeed elicited in these cases, and we show how using a scaling approach can appropriately weight these observations in the econometric model. Finally, we find that some respondents ignore attributes for protest-like reasons which essentially convey no information about preferences. We suggest that using the standard approach combined with weighting procedures and recoding of non-attendance statements conditional on the specific reasons for non-attendance could be more appropriate than the current standard way of taking stated non-attendance into account.
\end{abstract}

Keywords: choice experiment, attribute non-attendance, passive bounded rationality, rationally adaptive behaviour, error component logit model

\section{JEL Classifications:}

C25, C93, D12, Q22, Q26, Q51 


\section{Introduction}

Choice experiments (CE) are being increasingly used to measure the value of non-market goods and services within a wide range of different fields. CE involves estimating the values of non-market goods and services by observing the choices made by respondents between various attributes of a given good or service. An issue that has received much attention in the literature recently is attribute non-attendance. In order to decide between competing alternatives in choice sets in $\mathrm{CE}$, respondents might employ various attribute processing strategies and some of these could potentially lead to choice inconsistency (Sælensminde 2001).

One such strategy is attribute non-attendance, i.e. where respondents ignore one or more attributes of the alternatives when making their choices (Hensher et al. 2005a; Campbell et al. 2008; Hensher 2010). This implies that individuals do not make the assumed trade-offs between attributes and attribute levels (Rosenberger et al. 2003; and Gowdy and Mayumi 2001). In other words, this violates the assumption of compensatory behaviour, i.e. the continuity axiom which is a standard assumption in the neoclassical theoretical framework underlying CE. Since respondents showing such non-compensatory behaviour cannot be represented by the conventional utility function, the computation of marginal rates of substitution, and thus, the estimation of the willingness to pay (WTP) is prohibited (Campbell et al. 2008; Campbell et al. 2010; Lancsar and Louviere 2006; Lockwood 1996; Scarpa et al. 2008).

Recently, several studies have focused on unveiling how respondents process attributes, and in particular how attribute non-attendance affects results and how it should be accounted for in CE surveys (e.g. Scarpa et al. 2010; Hensher et al. 2005a; Campbell et al. 2008; Carlsson et al. 2010; Kaye-blake et al. 2009; Scarpa et al. 2009). While some of these studies identify attribute non-attendance by making use of a stated non-attendance (SNA) approach (Hensher et al. 2005a; Carlsson et al. 2010), others rely on analytical nonattendance (ANA) approaches (e.g. Scarpa et al. 2009; Campbell 2008). While the former identifies attribute non-attendance by directly asking respondents whether or not they have ignored attributes, the latter focuses on identifying non-attendance by use of analytical models rather than respondent statements. Based on simulation experiments Hoyos et al. (2010) show that the SNA approach may be more appropriate than the ANA approach ${ }^{1}$. In the present paper, we focus on the SNA approach.

Typically, in the SNA approach a respondent's stated non-attendance to a specific attribute entails that for this respondent the contribution to the likelihood function is set to zero for that particular attribute. The implicit assumption is that an observed choice essentially provides no information concerning the respondent's preferences for this attribute. This seems to have become a more or less standard approach of dealing with attribute nonattendance in CE. However, Campbell and Lorimer (2009) find evidence suggesting that even though respondents state that they have ignored an attribute, this might not be entirely true. Specifically, they find a discrepancy between the stated non-attendance responses and the attribute processing strategies picked up by an analytical model. Hence, the standard approach for accommodating attribute non-attendance by fixing the contribution to the likelihood function to zero may be inappropriate. Furthermore, this approach may not adequately take heterogeneous attribute processing strategies into account.

\footnotetext{
${ }^{1}$ In particular, Hoyos et al. (2010) find that the SNA approach provides unbiased welfare estimates in the case of uncorrelated errors, while the ANA approach fails to do so. However, when errors are correlated none of the two approaches are able to provide unbiased welfare estimates in all cases.
} 
Even though the majority of studies focusing on attribute non-attendance would suggest that taking non-attendance into account in one way or another is likely to improve model performance and potentially affect WTP estimates, the results in the literature are ambiguous. There is no general consensus on exactly how attribute non-attendance should be dealt with. However, it does seem that there is a general consensus that ignoring attribute non-attendance while maintaining the passive bounded rationality assumption implies potentially biased and erroneous welfare estimates and poor model performance. All studies investigating attribute non-attendance agree that respondents do ignore attributes to some extent. Hence, the pressing research question is no longer whether or not respondents ignore attributes, but rather exploring the underlying reasons why respondents ignore attributes, as underlined by both Carlsson et al. (2010) and Scarpa et al. (2010). The current paper aims to contribute to this research question.

Despite the great attention that attribute non-attendance has received in recent years, it seems that few have ventured into investigating the underlying behavioural mechanisms and decision processes. Though this has received some attention in the ANA approach (e.g. Hensher 2006), to the authors' knowledge it remains a largely unexplored area in the SNA approach. In order to identify and incorporate procedures for dealing with heterogeneous attribute processing strategies, we propose an extension of the SNA approach, focusing on the behavioural reasons underlying the statements of attribute non-attendance. Specifically, we find that some respondents act in accordance with the passive bounded rationality assumption since they ignore an attribute simply because it does not affect their utility. This is confirmed by the analytical model. Though we do not find significant evidence, we argue that excluding these genuine zero preferences from affecting the mean attribute parameter estimates, as the standard approach entails, could potentially bias results. Hence, we suggest recoding these non-attendance statements from ignored to non-ignored. Other respondents claim to have ignored attributes to simplify choices. However, we find that these respondents have actually not completely ignored attributes. We argue along the rationally adaptive behavioural model that preferences are indeed elicited in these cases, and we show how using a scaling approach can appropriately weight these observations in the econometric model. Finally, we find that some respondents ignore attributes for protest-like reasons which essentially convey no information about preferences despite the analytical model picking up some significant parameters. Our results suggest that using the standard approach in combination with weighting procedures as well as recoding of non-attendance statements conditional on the specific reasons for non-attendance, could be more appropriate than the current standard practice where all statements of attribute non-attendance are treated homogeneously. Furthermore, our results suggest that the SNA approach needs to be improved in order to better distinguish between non-attendance and low preferences.

The remainder of the paper is organized as follows. Section 2 provides a brief overview of the literature concerning attribute non-attendance and attribute processing strategies. Section 3 describes the data. Section 4 introduces the econometric specification of models used, while section 5 presents the results and the discussion. Finally, concluding remarks are presented in section 6 .

\section{Attribute non-attendance}

Two approaches to indentifying attribute non-attendance in CE have been suggested in the literature. While the SNA approach identifies attribute non-attendance by relying on directly asking respondents whether or not they have ignored attributes, the ANA approach focuses on identifying non-attendance by use of analytical models rather than respondent statements. The two approaches will be discussed in more detail below. 


\subsection{Stated non-attendance (SNA)}

Campbell et al. (2008) find that $36 \%$ of respondents state that they have ignored at least one of the attributes used in a survey considering improvements in rural landscape attributes in the republic of Ireland. Their analysis shows that accounting for attribute nonattendance behaviour by restricting the individual behaviour to zero for attributes stated to have been ignored results in better model fits. Furthermore, the magnitude of the WTP estimates of each attribute decrease, implying that disregarding attribute non-attendance behaviour in CE could potentially cause inflated WTP estimates. In a study covering three surveys concerning the value of Sweden's Environmental Quality Objectives, Carlsson et al. (2010) find that the majority of respondents exclude at least one attribute. However, contrary to the findings of Campbell et al. (2008) they find no significant change in WTP when accounting for non-attendance. Further, they find worse model fit for models where ignored parameters are restricted to zero than for models assuming passive bounded rationality.

Hensher et al. (2005a) examine the impact of ignoring specific attributes on the WTP for travel time savings based on supplementary information obtained from car commuters in Sydney. They find model fit to increase and WTP estimates for travel time savings to decrease when attribute non-attendance is accounted for by restricting ignored parameters to zero.

Campbell and Lorimer (2009) find that only $25 \%$ of their respondents take all the attributes into account during the choice tasks. With regard to model fit and WTP, they find that there is an improvement in model performance and the WTP have a lower magnitude when attribute processing strategies are detected. Hensher et al. (2007) find that around half of the total sample $(52 \%)$ attended to all attributes presented in the choice set. As opposed to Hensher et al. (2005a), they find that model fit as well as WTP values decrease in a model where ignored attributes are restricted to zero.

Puckett and Hensher $(2008 ; 2009)$ conducted a study revealing process heterogeneity in CE by using data from road freight transport providers and their customers in Sydney. They conclude that there is substantial heterogeneity in terms of attribute non-attendance behaviour across respondents as well as across choices, and taking this into account improves model fit slightly. They also find that when this behaviour is neglected, the WTP for travel time savings is potentially overestimated.

Hensher (2006) suggests that it may be beneficial to identify attribute non-attendance at the choice task level rather than at the overall choice task sequence level. Puckett and Hensher $(2008 ; 2009)$ are, to the authors' knowledge, the first to explicitly do this, though they do not focus much on this particular issue. In a survey focusing on the externalities of onshore wind power, Meyerhoff and Liebe (2009) target this issue and find evidence that non-attendance is indeed choice task specific. Comparing models that account for choice task non-attendance and so-called reconstructed serial non-attendance, they find the former to provide better model fit though not causing any significant differences in WTP estimates. Scarpa et al. (2010) continue this line of research in a study focusing on the value of alpine park management services. Specifically, they find marked improvements in model fit especially when accounting for non-attendance at the choice task level. They do not find a unidirectional change in WTP as it increases for some attributes while decreasing for others when accounting for non-attendance. They do, however, find that accounting for nonattendance at the choice task level generally implies smaller WTP estimates than in models where non-attendance is accounted for at the overall choice task level or not at all.

\subsection{Analytical non-attendance (ANA)}

Campbell (2008) examines how to analytically derive and incorporate discontinuous preferences in a $\mathrm{CE}$ survey concerning the existence value of rare and endangered fish 
species in Lough Melvin Catchment in Ireland. He uses a two-step estimation procedure: First, a two-class latent class model (LCM) is estimated where all the attribute coefficients in the first class are freely estimable, while the attribute coefficients in the second class are restricted to zero. The estimated class membership probabilities are then used to condition the attribute coefficients in an error component model in order to take attribute non-attendance into account. Comparing this model to a benchmark error component model where nonattendance is not accounted for, Campbell finds better model fit as well as significantly lower WTP estimates when attribute non-attendance is accounted for. In a more recent paper, Campbell et al. (2010) find similar results using a more flexible LCM model which takes all possible attribute non-attendance strategies into account. In contrast, Hensher and Rose (2009) find that taking attribute non-attendance behaviour into account leads to increasing WTP for travel time savings, though still slightly improving the fit of the model.

Scarpa et al. (2009) suggest two ways of handling attribute non-attendance in choice modelling. These are stochastic attribute selection and Bayesian estimation procedure. They also find improved goodness-of-fit when accounting for non-attendance, and they further argue that this leads to more appropriate estimates of the WTP. Similarly, Hensher and Greene (2010) specify a LCM and find a model with better fit when accounting for attribute process heterogeneity. However, they find that when the process heterogeneity is incorporated in the model, the WTP for travel time savings is higher than when using an MNL model that does not account for heterogeneity in attribute processing strategies.

Hole (2010) examines the impact of attribute non-attendance in CE designed to determine the preference of patients for primary care consultation. He finds a substantial improvement in model fit as well as overall reductions in WTP estimates when accounting for non-attendance using a so-called endogenous attribute attendance model.

\subsection{Attribute processing strategies}

DeShazo and Fermo, (2004) present two general attribute processing strategies: the passive bounded rationality model, and the rationally adaptive behavioural model. They define passive bounded rationality as respondents who consider all information in the choice set, but with increasing tendency of making mistakes as a result of increasing the complexity of the choice set, for instance by increasing the number of attributes and levels (DeShazo and Fermo, 2002). Opposed to this, the rationally adaptive behavioural model assumes that individuals realise that their limited cognition has positive opportunity costs. As a result, individuals process the attribute information to minimise the cost and to maximise the benefit. In relation to $\mathrm{CE}$, this could imply that respondents would ignore more attributes when the total number of attributes used is increased, as found by e.g. Hensher (2006). If an individual behaves as prescribed by the rationally adaptive model, it poses a problem for the researcher to use the standard model assuming that individuals consider all attribute information in the choice sets.

\section{Data and Method}

\subsection{Data description}

The conducted CE survey is intended for assessing the value of fishing site characteristics. Specifically, the aim is to uncover German tourists' preferences for recreational fishing at different hypothetical fishing sites when on vacation in Denmark. Six different fishing site quality attributes, each with three different levels, were used in the survey. Furthermore, a cost attribute with six different levels was included. All attributes and levels are presented in table 1 and were identified using extensive focus group testing and a pilot study. 
Table 1: Attributes and levels used in the CE survey

\begin{tabular}{ll}
\hline Attributes & Levels (coding) \\
\hline Catch opportunity (Catch) & Low (Catch0) \\
& Medium (Catch1) \\
& High (Catch2) \\
& Many small and few large fish (Size0) \\
Fish size (Size) & A mix of smaller and larger fix (Size1) \\
& Large fish and the possibility of record-breaking large fish (Size2) \\
& Small (Nature0) \\
Nature experience (Nature) & Varied (Nature1) \\
& Big (Nature2) \\
& Unclear and appears to be polluted (Quality0) \\
Quality of the fishing water & Reasonably clean and clear (Quality1) \\
(Quality) & Clean and clear (Quality2) \\
& $<4$ km (Distance0) \\
Distance from accommodation & 4-20 km (Distance1) \\
to site (Distance) & $>20$ km (Distance2) \\
& None (Number0) \\
Number of other anglers at site & Few (Number1) \\
(Number) & Many (Number2) \\
& $7,15,25,40,75,140 €$ \\
Cost of one-day fishing license \\
(Cost)
\end{tabular}

An advanced awareness treatment similar to that of Bateman et al. (2008) is used in order to facilitate learning processes and give respondents a grasp of the attributes and levels before the actual preference eliciting choice sets are presented. Each choice set consists of two experimentally designed alternatives and a zero-cost 'none-of-these' opt-out alternative.

The experimental design used is a D-efficient Bayesian updated fractional factorial design resulting in 18 different choice sets in total. The updated design is based on results from a pilot study with 103 respondents. The total sample of respondents was split into three blocks since the focus group interviews revealed that 18 choice sets per respondents were too many. Hence, each respondent evaluated six different choice sets which was found to be a more suitable number of choice tasks per respondent. Respondents were sampled from a prerecruited internet panel on the condition that they could be classified as recreational fishers since this was considered the relevant target population. A total of 1006 respondents replied to the questionnaire, of which 38 were identified as protesters ${ }^{2}$, thus leaving an effective sample of 968 respondents for the analysis.

\footnotetext{
${ }^{2}$ Protesters were identified using a follow-up question given to respondents who chose the zero-cost opt-out alternative in all six choice sets. Those stating reasons such as "I don't want to pay for better fishing in Denmark", "I don't believe that the changes shown will take place", "The Danes must pay for better fishing in Denmark themselves", "I would never engage in recreational fishing in Denmark", or "I don't know" were classified as protesters and excluded from the analysis in line with Morrison et al. (2000) and Meyerhoff and Liebe (2006).
} 


\subsection{Identification of attribute non-attendance}

In the present study, information is obtained from supplementary questions asking respondents whether they ignore some specific attributes or not ${ }^{3}$. In addition, the reasons why respondents ignore attributes of alternatives in the choice set are obtained from the subsequent follow-up questions displayed in figure 1. This follow-up question is asked for each attribute that is stated to have been ignored.

\begin{tabular}{|l|c|}
\hline What was your primary cause for ignoring this attribute? (Tick only one) & $\begin{array}{c}\text { Catch } \\
\text { opportunity }\end{array}$ \\
\hline It is not important to me & $\square$ \\
\hline It made it easier to choose between the alternatives & $\square$ \\
\hline The levels for the attribute were unrealistically high/low & $\square$ \\
\hline I don't think that this attribute should be weighed against the others (i.e. no trade-off) & $\square$ \\
\hline Don't know & $\square$ \\
\hline
\end{tabular}

Figure 1: Example of follow-up question regarding why respondents ignored specific attributes - in this case for the attribute 'Catch opportunity'.

\section{Econometric specification}

The model applied in the parametric analysis of responses is a mixed logit model which can be derived in a number of different ways (see Train 2003; Hensher and Greene 2003). In the present case, a model formulation which incorporates an error component was found suitable ${ }^{4}$.

Following Scarpa et al. (2005) an Alternative Specific Constant (ASC) is specified for the status quo alternative in order to capture the systematic component of a potential status quo effect. Furthermore, an error component additional to the usual Gumbel-distributed error term is incorporated in the model to capture any remaining status quo effects in the stochastic part of utility. The error component, $\mu$, which is implemented as an individual-specific zeromean normally distributed random parameter, is assigned exclusively to the two non-status

\footnotetext{
${ }^{3}$ Respondents were only asked whether they ignored non-price attributes. Hence, we have no information about price attribute non-attendance. Despite having to assume full attendance to the price attribute, the data can still be considered suitable for the purpose at hand, namely exploring and incorporating the behavioural reasons underlying non-attendance, with a focus on the quality attributes. For the same reason, we only briefly deal with impact on WTP. As WTP calculations entail dividing a quality attribute with the price attribute, we believe that non-attendance information about both these attributes are necessary to assess the impact on WTP of different behavioural decision strategies underlying non-attendance. Hence, we leave this for future research.

${ }^{4}$ Employing random parameter logit (RPL) models might be more informative if one aims for knowledge about heterogeneity in preferences. However, we have a number of reasons for choosing the error component logit (ECL) model instead. We did initially specify RPL for the first four models, but ran into identification problems in model 5 due to a very large number $(>100)$ of parameters to be estimated. Comparing ECL and RPL results for the four first models, we found no differences in the overall conclusions reached. Furthermore, the model fit of the RPL and ECL models were almost identical. Even though the RPL models picked up heterogeneous preferences for some of the quality attributes, we found only minor impacts of non-attendance and underlying reasons on this heterogeneity in terms of slightly increasing numbers of significant standard deviation estimates when moving from model 1 to 4 . This might suggest that accounting for non-attendance and underlying reasons is important in order to properly identify heterogeneous preferences. However, this tendency was not very strong, so we leave this to be investigated more rigorously in future work. Hence, we have chosen the ECL model for reasons of simplicity and to ensure comparability across all five models.
} 
quo alternatives. By specifying a common error component across these two alternatives, correlation patterns in utility over these alternatives is induced. Thus, it captures any additional variance associated with the cognitive effort of evaluating experimentally designed hypothetical alternatives (Greene and Hensher 2007; Scarpa et al. 2007; Scarpa et al. 2008). This results in the following general utility structure:

$$
U_{n t j}=\left\{\begin{array}{l}
V\left(x_{n t j}, \beta, \mu\right)+\varepsilon_{n t j}, j=1,2 ; \\
V\left(A S C, x_{n t j}, \beta\right)+\varepsilon_{n t j}, j=S Q
\end{array}\right.
$$

where the indirect utility, $V$, is a function of the vector of explanatory variables, $x_{n j t}$, and associated parameters, $\beta$. For the two experimentally designed policy alternatives, the common error component $\mu$ enters the indirect utility function, while it is restricted to zero for the status quo alternative. The unobserved error term $\varepsilon_{n t j}$ is assumed Gumbel-distributed. The individuals are denoted by $n$, while $j$ is the alternative and $t$ is the choice set. The probability of individual $n$ choosing alternative $k$ out of $j$ alternatives can be defined by the multinomial logit model:

$$
P_{n t k}=\frac{e^{\lambda \beta^{\prime} x_{n t k}}}{\sum_{j}^{J} e^{\lambda \beta^{\prime} x_{n i j}}}
$$

where $\beta^{\prime}$ is a vector of all betas, $\lambda$ is the scale parameter which is typically normalised to 1 (the ASC and error terms from eq. (1) are left out of the equation for simplicity). Following Train (2003) and Scarpa et al. (2005), the probabilities of the error component logit (ECL) model can be described as integrals of the standard multinomial logit function evaluated at different $\mu$ 's with a density function as the mixing distribution. Furthermore, this specification can be generalised to allow for repeated choices by the same respondent, i.e. a panel structure, by letting $z$ be a sequence of alternatives, one for each choice occasion, $z$ $=\left\{z_{1}, \ldots, z_{T}\right\}$. Thus, the error component coefficients may vary over people but are constant over the $T$ choice occasions for each individual. The marginal choice probability then becomes:

$$
P_{n k z}=\int\left(\prod_{t=1}^{T}\left[\frac{e^{\lambda \beta^{\prime} x_{n k k}}}{\sum_{j}^{J} e^{\lambda \beta^{\prime} x t j}}\right]\right) \phi\left(\mu \mid 0, \sigma^{2}\right) d \mu
$$

where $\phi\left(\mu \mid 0, \sigma^{2}\right)$ is the normal density distribution function for $\mu$, with mean 0 and covariance $\sigma^{2}$.

Based on the above general econometric specification, we specify five different models. In all models we use a panel structure by assuming $\mu$ to be fixed across the entire sequence of choices made by the same respondent. The first three models are essentially similar to those specified in Campbell and Lorimer (2009), though we employ an ECL model and not a random parameter logit model. Model 1 involves estimation of the ECL model without accounting for attribute non-attendance. Model 2, however, restricts ignored attribute parameters to zero. This represents the standard way of dealing with attribute non-attendance in the SNA approach. Inspired by Campbell and Lorimer (2009), model 3 estimates two coefficients for each attribute as separate parameters estimated depending on whether the attribute is considered or ignored. In models 1 to 3 , we use the common normalisation of $\lambda$ to unity (Train 2003, Scarpa et al. 2003), implying that unexplained variance is assumed 
uniform across responses. Inspired by Sælensminde (2001) and Campbell et al. (2008), in model 4 we relax this assumption and allow for potential differences in scale depending on whether or not attributes have been ignored, and, in particular, depending on the reasons why they have been ignored. A general parameterisation of the scale function is (Hensher et al. 2005b; Lundhede et al. 2009; Scarpa et al. 2003):

$$
\lambda=\exp \left(\gamma_{w} Z_{n w}\right)=\frac{\pi}{\sqrt{\operatorname{var}\left(\varepsilon_{n}\right)} \sqrt{6}}
$$

where $Z_{n w}$ is a vector of covariates associated with the five specific reasons stated for ignoring attributes (the elements in $Z$ are indexed by $w$ ), and $\gamma_{w}$ is a row-vector of the corresponding scale function parameters. The exponential form of the scale function ensures nonnegative estimates of model variance, as the scale is inversely proportional to the standard deviation of the unobserved component, $\varepsilon_{n}$. In model 5 we elaborate on model 3 by estimating a full set of preference parameters based on the five different reasons for ignoring attributes. This is in contrast to model 3, where the estimation only was base on the aggregate group of ignored attributes. We essentially disintegrate each attribute parameter for the ignored attributes into five groups based on the stated reasons for ignoring. Hence, we estimate parameter estimates for all attributes for all these five groups as well as the group paying attention to the all attributes.

\section{Results and discussion}

\subsection{Summary of attribute attendance and non-attendance}

Table 2 shows that out of the 968 respondents, 343 have stated that they did not ignore any attributes in the choice sets. Thus, these respondents apparently adhere to the continuity axiom as commonly assumed in CE. However, the remaining 625 respondents state that they have not considered all attributes when making their choices, implying that these respondents have discontinuous preferences to some extent. The rather high rate of nonattendance in the survey is in correspondence with the general finding in the existing literature dealing with attribute non-attendance, namely that it is clearly a non-negligible proportion of respondents who do not adhere to the standard axiomatic assumptions in CE. It is not uncommon to find that less than half of the respondents actually consider all attributes (e.g. Campbell and Lorimer 2009; Carlsson et al., 2010; Hensher et al., 2007). Therefore, maintaining the passive bounded rationality assumption relying on individuals considering all attribute information displayed in the choice set may result in biased taste parameters.

Table 2: Number of respondents who ignored one or several attributes

\begin{tabular}{lcc}
\hline No. of attributes ignored & No. of respondents & \% of respondents \\
\hline Zero & 343 & 35.4 \\
One & 222 & 22.9 \\
Two & 236 & 24.4 \\
Three & 121 & 12.5 \\
Four & 33 & 3.4 \\
Five & 5 & 0.5 \\
Six & 8 & 0.8 \\
Total & 968 & 100 \\
\hline
\end{tabular}

5.2 Why do respondents ignore attributes when processing choice task information? 
Table 3 shows that the most ignored attribute is the Size attribute which was ignored by 252 respondents while the least ignored is the Quality attribute which was ignored by 171 respondents. According to Carlsson et al. (2010), this might suggest that anglers in general have stronger preferences for the quality of the water than for the size of the fish they might catch when going on a fishing trip.

Table 3: Stated reasons for attribute non-attendance.

\begin{tabular}{|c|c|c|c|c|c|c|}
\hline \multirow[b]{2}{*}{ Reason for ignoring } & \multicolumn{6}{|c|}{ No. (and \%) of times attribute is ignored } \\
\hline & Catch & Size & Nature & Quality & Distance & Number \\
\hline 1.It is not important to me & $\begin{array}{c}54 \\
(25 \%)\end{array}$ & $\begin{array}{c}88 \\
(35 \%)\end{array}$ & $\begin{array}{c}20 \\
(9 \%)\end{array}$ & $\begin{array}{c}13 \\
(8 \%)\end{array}$ & $\begin{array}{c}84 \\
(39 \%)\end{array}$ & $\begin{array}{c}52 \\
(28 \%)\end{array}$ \\
\hline $\begin{array}{l}\text { 2. It made it easier to choose } \\
\text { between alternatives }\end{array}$ & $\begin{array}{c}100 \\
(46 \%)\end{array}$ & $\begin{array}{c}108 \\
(43 \%)\end{array}$ & $\begin{array}{c}120 \\
(57 \%)\end{array}$ & $\begin{array}{c}101 \\
(59 \%)\end{array}$ & $\begin{array}{c}75 \\
(35 \%)\end{array}$ & $\begin{array}{c}87 \\
(46 \%)\end{array}$ \\
\hline $\begin{array}{l}\text { 3. The levels for the attribute } \\
\text { were unrealistically high/low }\end{array}$ & $\begin{array}{c}26 \\
(12 \%)\end{array}$ & $\begin{array}{c}20 \\
(8 \%)\end{array}$ & $\begin{array}{c}28 \\
(13 \%)\end{array}$ & $\begin{array}{c}29 \\
(17 \%)\end{array}$ & $\begin{array}{c}19 \\
(9 \%)\end{array}$ & $\begin{array}{c}15 \\
(8 \%)\end{array}$ \\
\hline $\begin{array}{l}\text { 4. I don't think that this } \\
\text { attribute should be weighed } \\
\text { against the others }\end{array}$ & $\begin{array}{c}26 \\
(12 \%)\end{array}$ & $\begin{array}{c}27 \\
(11 \%)\end{array}$ & $\begin{array}{c}28 \\
(13 \%)\end{array}$ & $\begin{array}{c}20 \\
(12 \%)\end{array}$ & $\begin{array}{c}30 \\
(14 \%)\end{array}$ & $\begin{array}{c}26 \\
(14 \%)\end{array}$ \\
\hline 5. Do not know & $\begin{array}{c}11 \\
(5 \%)\end{array}$ & $\begin{array}{c}9 \\
(4 \%)\end{array}$ & $\begin{array}{c}16 \\
(8 \%)\end{array}$ & $\begin{array}{c}8 \\
(5 \%)\end{array}$ & $\begin{array}{c}5 \\
(2 \%)\end{array}$ & $\begin{array}{c}9 \\
(5 \%)\end{array}$ \\
\hline Total & 217 & 252 & 212 & 171 & 213 & 189 \\
\hline
\end{tabular}

Looking at the stated reasons for ignoring attributes the most often stated reason is 'It made it easier to choose between alternatives'. This reflects a simplifying heuristic which is in support of the rationally adaptive behaviour model. This model recognises that attending to all information in a choice set can be very cognitively demanding. If respondents realise this they may employ an attribute processing strategy that maximises the benefits and minimises the cognitive costs associated with processing the information, for instance by ignoring 'difficult' attributes (DeShazo and Fermo, 2004). In particular, for the Nature and Quality attributes this reason for non-attendance is stated $57 \%$ and $59 \%$ of the times respectively, while the number for all other attributes is at or below $46 \%$. This suggests that the cost of information processing in terms of cognitive load is higher for these attributes, which in a sense have more non-market good characteristics than other more 'market-like' attributes such as Distance and Size. This is in line with Campbell et al. (2008), who note that especially in the case of complex and unfamiliar ecological and environmental goods, the choice tasks respondents are expected to perform require a significant cognitive effort. Considering the fact that this reason for ignoring particular attributes has been stated 591 times in total ${ }^{5}$, it is evident that including such an amount of observations in a standard CE model assuming passive bounded rationality is likely to bias results.

The second-most stated reason for ignoring specific attributes is that 'It is not important for me'. We interpret this as expressing a genuine preference. As Carlsson et al.

\footnotetext{
${ }^{5}$ Notice that respondents ignoring more than one attribute could potentially state the same reason several times, i.e. for each ignored attribute, this does not mean that 591 different respondents have stated this reason. Also notice that since the non-attendance question considered all six choices made by a respondent, this implies that a total of 3546 preference eliciting attribute observations are affected by this reason.
} 
(2010) note, respondents may state that they ignore an attribute simply because they do not have a WTP for the proposed change in that attribute. In other terms, this attribute does not affect their utility. Ryan et al. (2009) find that several respondents ignore attributes simply because the attributes are not relevant to them. Since this reason for ignoring an attribute is in line with the standard assumptions under the passive bounded rationality model, there is no reason to treat these observations any differently than attributes which have not been ignored. Considering that this reason is stated in as many as 316 occasions in the present study, this finding has important implications. When identifying non-attendance behaviour using the SNA approach, there is no distinguishing between the underlying reasons. The standard way of treating non-attendance is to set the contribution to the likelihood function to zero for observations where non-attendance is identified. Hence, these observations, where respondents' state that they have ignored an attribute - and the underlying reason is that the attribute does not affect their utility - would be treated as if they were in violation of the passive bounded rationality model even though this is not the case. They would essentially be excluded from the preference model implying a potential loss of efficiency in modelling terms, and furthermore, results from the preference model might be biased. More precisely, attribute parameter mean estimates might be under or overestimated since a number of essentially genuine zero preferences have been excluded from the model ${ }^{6}$. Thus, our results suggest that when using the SNA approach, it is important to ask additional debriefing questions concerning the reasons underlying non-attendance statements.

The third reason for non-attendance which is stated less frequently than the first two mentioned, is 'The levels for the attribute were unrealistically high/low'. This statement could reflect protest-like behaviour in the sense that the respondent does not believe the attribute changes described and, therefore, decides to ignore the attribute. Protest behaviour is typically identified at the choice set sequence level by asking repeated zero-bidders to state their reasons for choosing a zero-priced alternative in all choice sets. The researcher then classifies the reasons as either valid or protest. A potential reason which is typically classified as a protest bid is 'The scenario/attributes/levels were unrealistic' (Jorgenson et al. 1999). All choices from respondents identified as protesters are typically excluded from the analysis (Morrison et al. 2000). In our study we have done this. However, there is no a priori reason why protest behaviour could not be equally present at the attribute level to the extent that the respondent does not protest against the overall survey but rather against specific attributes in the survey. Our results suggest that indeed there may be some respondents, who protest against particular attributes while accepting others. This type of 'refusing to play [a certain part of] the game' (Mitchell and Carson 1989, p.166) protest behaviour is clearly not in line with the standard passive bounded rationality assumptions in CE. Another interpretation of the 'The levels for the attribute were unrealistically high/low' statement could be that it reflects the satisficing heuristic described by Payne et al. (1992). If the respondent uses certain cut-off levels to decide whether or not to ignore an attribute this might be reflected in an 'unrealistically high/low' statement. Yet another potential decision making strategy in play here could be the elimination by aspects heuristic (Payne et al. 1992). If the respondent finds that an important attribute does not meet a certain minimum acceptable level, the respondent might ignore this attribute and focus on other important attributes (which meet their minimum acceptable levels) instead. If the minimum acceptable level of an important attribute is not met, it seems likely that the respondent might reason his attribute nonattendance with the 'unrealistically low' level of the attribute. Of course, no matter which of

\footnotetext{
${ }^{6}$ This has been investigated in our data, and the results show that the mean parameter estimates were reduced when these genuine zero preferences were included, though not statistically significant.
} 
these two decision making strategies underlie respondents' decision to ignore attributes, they are clearly in violation of the passive bounded rationality assumptions.

The fourth reason which has been stated $12-14 \%$ of the times attributes have been ignored is 'I don't think that this attribute should be weighed against the others'. As above, this could be interpreted as protest behaviour since this type of argument is often used to identify zero-protesters at the choice task sequence level. Another interpretation could be that this statement reflects a lexicographic heuristic being used (Payne et al. 1992). If the respondents make their choices based solely on the most important attribute, it seems likely that they might ignore the remaining attributes and for each of these state the above reason. However, the present data does not support this interpretation since none of the respondents ignoring five or six attributes stated solely this reason for ignoring the attributes. Whether or not this reason is associated with protest behaviour or a lexicographic heuristic, the implication is that respondents do not actually make the required trade-offs for these attributes as assumed in the passive bounded rationality model.

Finally, there is a relatively low number of 'Do not know' statements which we cannot really interpret. Since we have used a closed form for the follow-up question with four different answer categories besides the 'Do not know' option, it is not unlikely that some respondents have ignored attributes for reasons that are not covered in these categories. If so, respondents might state a 'Do not know' simply because their reasons do not fit into any of the other categories. We are not able to explore this further in the current dataset. Of course, it might also be that respondents simply do not know why they ignored the attributes. In any case it is not possible to conjecture whether or not this reason is in accordance with the passive bounded rationality assumption. Considering the rather low number of observations here, a conservative approach of excluding them from the preference model might be a practical solution.

\subsection{Estimation results}

Tables 4 and 5 display the estimation results obtained for the five models. All models are estimated using the software package BIOGEME (Bierlaire, 2003). In the simulated maximum likelihood estimation 300 Halton draws were used as this was found to be a sufficient number for results to stabilise. For the three-level attributes, using dummy coding we estimate parameters for levels 1 and 2 relative to level 0 as specified in table 1 .

\subsubsection{The SNA approach so far}

Models 1, 2 and 3 are intended to represent the modelling approaches that have been used in the SNA literature until now. In all three models all of the estimated attribute parameters are statistically significant at or above the $95 \%$ significance level, with the exception of Size1 in model 2. Model 1 shows that the most important attribute is the quality of the fishing water (Quality), followed by catch opportunity (Catch) and nature experience (Nature). Two of the six fishing site attributes have negative signs. These are distance from accommodation to site (Distance) and number of other anglers at the site (Number). This is in accordance with the a priori expectation that as the distance from the accommodation to the fishing site increases, the utility decreases due to the costs of transportation both in terms of time and money. Furthermore, increasing the number of anglers at the fishing site could cause congestion which is not surprisingly associated with decreasing utility. Similarly, the cost parameter is highly significant in all models with a negative sign which is in accordance with predictions based on standard economic theory.

In all models, the status quo alternative specific constant $\left(\mathrm{ASC}_{\mathrm{sq}}\right)$ is statistically significant with a negative sign which indicates that individuals ceteris paribus favour the proposed experimentally designed alternatives over the status-quo alternative 'none of these'. 
Assigning a behavioural interpretation to this estimate is difficult, but one explanation might be that respondents have perceived the 'none of these' status-quo alternative as implying that they would not go fishing. In that case this estimate could imply that respondents would rather go fishing than not, regardless of the fishing site characteristics. The error component $\mu_{n}$ which allows for correlation in error structure across the two experimentally designed alternatives is also highly significant. This suggests that a more flexible substitution pattern than the one assumed in a standard multinomial logit model is appropriate here. The variance for the unobserved part of the utility for the 'none of these' alternative is fixed to $\pi^{2} / 6$, while the variance associated with the two experimentally designed alternatives is $\pi^{2} / 6+2.23^{2}$ (for model 1), suggesting the presence of heteroskedasticity. This finding corresponds to the findings in Scarpa et al. (2007) and Hess and Ross (2009).

Comparing results obtained under the standard way of dealing with non-attendance (model 2) to the results obtained in the benchmark model where non-attendance is not taken into account (model 1), we find only minor differences. Besides the fact that the Size1 parameter is insignificant in model 2, the overall conclusions reached in these two models are alike. Similar to Carlsson et al. (2010), we see a decrease in model fit. Even though most others have found increases in model fit when accounting for non-attendance (e.g. Campbell et al. 2008; Hensher et al. 2005a; Puckett and Hensher 2008), considering the amount of observations which are essentially excluded from contributing to the likelihood it may not be surprising that we see this decrease. For some attributes it is up to $25 \%$ of the observations contributing to the likelihood in model 1 which are excluded from doing so in model 2. Ceteris paribus, decreasing the number of observations in a dataset would be expected to reduce the explanatory power of the model.

Campbell and Lorimer (2009) argue that it is important to examine whether the standard approach of fixing ignored attributes to zero in the likelihood function is appropriate or not. In model 3, separate parameters are estimated for each attribute depending on whether the attribute is considered or ignored. If indeed the estimates for the ignored parameters are not significantly different from zero, the standard approach would be appropriate. However, we find that nine out of 12 estimates are significantly different from zero which suggests that assigning a zero value to these parameters may not be feasible. Furthermore, they have the same signs and largely the same relative magnitudes as we find for the parameters based on considered attributes. This is in line with Campbell and Lorimer's (2009) findings that respondents who state that they have ignored an attribute have not actually ignored it completely. Carlsson et al. (2010) also conclude that one should not blindly trust these statements of non-attendance. Of course, the really important question here is whether or not the significant estimates obtained from attributes which respondents claim they have ignored, can be given a meaningful behavioural interpretation. Or maybe even more interesting; do the estimates represent preferences? Carlsson et al. (2010) suggest that rather than ignoring attributes completely, respondents might simply put less weight on attributes they claim to have ignored. They base this on the fact that the most often ignored non-monetary attributes also receives the lowest preference ranking in the estimated utility model. Our results confirm this tendency. The Size attribute which is the most ignored attribute in our survey is consistently the numerically least preferred attribute across all estimated models. The most preferred attribute is Quality which at the same time is also the least ignored attribute. This might serve as an indication that indeed there could be some relation between respondents' preferences and the information that the analytical model is able to extract from seemingly ignored attributes. This suspicion is reinforced by the fact that when the Quality attribute is ignored, the reason is most often (59\%) that 'It made it easier to choose between alternatives'. In other words, the non-attendance question in some instances seems to pick up low preferences rather than actual ignoring of attributes. Carlsson et al. (2010) note that more 
knowledge about why an attribute is ignored is necessary, in order to explore this important research area in more detail and to more adequately take attribute processing strategies into account. Models 4 and 5 serve this purpose.

Table 4: Results from error component logit models 1 to 4.

\begin{tabular}{|c|c|c|c|c|}
\hline $\begin{array}{l}\text { Parameter } \\
\text { estimates }\end{array}$ & $\begin{array}{c}\text { Model } 1 \\
\text { Mean (Std.err.) }\end{array}$ & $\begin{array}{c}\text { Model } 2 \\
\text { Mean (Std.err.) }\end{array}$ & $\begin{array}{c}\text { Model } 3 \\
\text { Mean (Std.err.) }\end{array}$ & $\begin{array}{c}\text { Model } 4 \\
\text { Mean (Std.err.) }\end{array}$ \\
\hline Cost & $-0.0143 *(0.000763)$ & $-0.0132 *(0.000706)$ & $-0.0143 *(0.000765)$ & $-0.0143 *(0.000764)$ \\
\hline ASC & $-1.04 *(0.119)$ & $-1.27 *(0.110)$ & $-1.02 *(0.119)$ & $-1.03 *(0.119)$ \\
\hline$\mu_{n}$ & $2.23 *(0.108)$ & $2.33 *(0.110)$ & $2.23 *(0.107)$ & $2.24 *(0.108)$ \\
\hline \multicolumn{5}{|l|}{ Considered } \\
\hline Number2 & $-0.275^{*}(0.0538)$ & $-0.215^{*}(0.0582)$ & $-0.255^{*}(0.0609)$ & $-0.271 *(0.0524)$ \\
\hline Number1 & $-0.261 *(0.0543)$ & $-0.263 *(0.0588)$ & $-0.262 *(0.0608)$ & $-0.255^{*}(0.0535)$ \\
\hline Catch2 & $0.615^{*}(0.0539)$ & $0.578 *(0.0595)$ & $0.623 *(0.0606)$ & $0.596 *(0.0539)$ \\
\hline Catch1 & $0.379 *(0.0547)$ & $0.353 *(0.0594)$ & $0.387 *(0.0616)$ & $0.366^{*}(0.0542)$ \\
\hline Distance2 & $-0.483 *(0.0596)$ & $-0.526 *(0.0638)$ & $-0.574 *(0.0668)$ & $-0.468 *(0.0599)$ \\
\hline Distance 1 & $-0.218 *(0.0460)$ & $-0.287 *(0.0512)$ & $-0.270 *(0.0520)$ & $-0.212 *(0.0455)$ \\
\hline Nature2 & $0.470 *(0.0462)$ & $0.459 *(0.0511)$ & $0.490 *(0.0520)$ & $0.451 *(0.0474)$ \\
\hline Nature1 & $0.153 *(0.0534)$ & $0.155 *(0.0585)$ & $0.161 *(0.0603)$ & $0.151 *(0.0516)$ \\
\hline Quality2 & $0.816 *(0.0611$ & $0.699 *(0.0630)$ & $0.791 *(0.0668)$ & $0.797 *(0.0616)$ \\
\hline Quality1 & $0.474 *(0.0581)$ & $0.373 *(0.0605)$ & $0.471 *(0.0634)$ & $0.465^{*}(0.0581)$ \\
\hline Size2 & $0.238 *(0.0550)$ & $0.130 *(0.0618)$ & $0.205^{*}(0.0640)$ & $0.236^{*}(0.0532)$ \\
\hline Size1 & $0.113 *(0.0496)$ & $0.026(0.0561)$ & $0.0627 *(0.0579)$ & $0.113 *(0.0482)$ \\
\hline \multicolumn{5}{|l|}{ Ignored } \\
\hline Number2 & & & $-0.371 *(0.0994)$ & \\
\hline Number1 & & & $-0.254 *(0.111)$ & \\
\hline Catch2 & & & $0.594 *(0.101)$ & \\
\hline Catch1 & & & $0.350 *(0.105)$ & \\
\hline Distance2 & & & $-0.175(0.0991)$ & \\
\hline Distance1 & & & $-0.0357(0.0852)$ & \\
\hline Nature2 & & & $0.422 *(0.0949)$ & \\
\hline Nature1 & & & $0.152(0.102)$ & \\
\hline Quality2 & & & $0.954 *(0.119)$ & \\
\hline Quality1 & & & $0.506 *(0.114)$ & \\
\hline Size2 & & & $0.333 *(0.0932)$ & \\
\hline Size1 & & & $0.253 *(0.0885)$ & \\
\hline$\lambda$ considered & & & & 1.00 (fixed) \\
\hline$\lambda_{\text {ignored_reason } 1}$ & & & & $0.007 *(0.00203)$ \\
\hline$\lambda_{\text {ignored_reason } 2}$ & & & & $0.202 *(0.198)$ \\
\hline$\lambda_{\text {ignored_reason } 3}$ & & & & $0.433(0.633)$ \\
\hline$\lambda_{\text {ignored_reason } 4}$ & & & & $0.161 *(0.266)$ \\
\hline$\lambda_{\text {ignored reason } 5}$ & & & & $0.589(0.850)$ \\
\hline Final LL & -5236.56 & -5305.09 & -5226.74 & -5235.82 \\
\hline Adj. rho-squared & 0.177 & 0.166 & 0.177 & 0.176 \\
\hline Observations & 5808 & 5808 & 5808 & 5808 \\
\hline
\end{tabular}

Note: * indicates statistical significance at or above the $95 \%$ level, The estimated scale parameters are transformed from the exponential form in accordance with equation 4 and Train (2003), and moreover tested relative to the scale parameter for considered attributes, which is normalised to one. 
Table 5: Results from error component logit model 5

\begin{tabular}{|c|c|c|c|c|c|c|}
\hline \multirow[b]{2}{*}{ Parameter estimates } & \multirow[b]{2}{*}{ Considered } & \multicolumn{5}{|c|}{ Ignored } \\
\hline & & Reason 1 & Reason 2 & Reason 3 & Reason 4 & Reason 5 \\
\hline \multirow[t]{2}{*}{ Number2 } & $-0.253^{*}$ & -0.324 & $-0.356^{*}$ & -0.305 & $-0.549^{*}$ & -0.468 \\
\hline & $(0.0614)$ & $(0.187)$ & $(0.150)$ & $(0.257)$ & $(0.270)$ & $(0.487)$ \\
\hline \multirow[t]{2}{*}{ Number1 } & $-0.261^{*}$ & -0.166 & -0.137 & -0.137 & -0.463 & $-2.32 *$ \\
\hline & $(0.0614)$ & $(0.223)$ & $(0.150)$ & $(0.398)$ & $(0.306)$ & $(0.652)$ \\
\hline \multirow[t]{2}{*}{ Catch2 } & $0.637^{*}$ & 0.259 & $0.846^{*}$ & 0.55 & 0.395 & $0.763 *$ \\
\hline & $(0.0611)$ & $(0.184)$ & $(0.141)$ & $(0.341)$ & $(0.307)$ & $(0.350)$ \\
\hline \multirow[t]{2}{*}{ Catch1 } & $0.394^{*}$ & 0.216 & $0.456^{*}$ & $0.833 *$ & -0.16 & 0.069 \\
\hline & $(0.0625)$ & $(0.181)$ & $(0.158)$ & $(0.343)$ & $(0.242)$ & $(0.561)$ \\
\hline \multirow[t]{2}{*}{ Distance2 } & $-0.583^{*}$ & 0.084 & -0.272 & -0.55 & -0.334 & -0.871 \\
\hline & $(0.0672)$ & $(0.151)$ & $(0.168)$ & $(0.391)$ & $(0.190)$ & $(0.481)$ \\
\hline \multirow[t]{2}{*}{ Distance1 } & $-0.284 *$ & 0.088 & -0.099 & 0.049 & -0.219 & $-1.45^{*}$ \\
\hline & $(0.0523)$ & $(0.121)$ & $(0.152)$ & $(0.345)$ & $(0.207)$ & $(0.469)$ \\
\hline \multirow[t]{2}{*}{ Nature2 } & $0.49 *$ & -0.047 & $0.47 *$ & -0.015 & $0.931 *$ & 0.733 \\
\hline & $(0.0525)$ & $(0.263)$ & $(0.120)$ & $(0.256)$ & $(0.293)$ & $(0.414)$ \\
\hline \multirow[t]{2}{*}{ Nature1 } & $0.169^{*}$ & -0.126 & 0.176 & -0.079 & 0.508 & 0.397 \\
\hline & $(0.0607)$ & $(0.293)$ & $(0.136)$ & $(0.241)$ & $(0.307)$ & $(0.430)$ \\
\hline \multirow[t]{2}{*}{ Quality2 } & $0.791 *$ & $0.84^{*}$ & $0.961^{*}$ & $1.45^{*}$ & 0.429 & 0.768 \\
\hline & $(0.0674)$ & $(0.272)$ & $(0.142)$ & $(0.375)$ & $(0.426)$ & $(0.677)$ \\
\hline \multirow[t]{2}{*}{ Quality1 } & $0.474 *$ & 0.612 & $0.41^{*}$ & $1.08^{*}$ & 0.035 & 0.844 \\
\hline & $(0.0639)$ & $(0.387)$ & $(0.140)$ & $(0.307)$ & $(0.327)$ & $(0.478)$ \\
\hline \multirow[t]{2}{*}{ Size2 } & $0.201 *$ & 0.079 & $0.451^{*}$ & $0.607^{*}$ & 0.551 & 0.118 \\
\hline & $(0.0645)$ & $(0.147)$ & $(0.142)$ & $(0.289)$ & $(0.352)$ & $(0.403)$ \\
\hline \multirow[t]{2}{*}{ Size1 } & 0.062 & 0.138 & 0.231 & 0.249 & $0.612 *$ & $0.632 *$ \\
\hline & $(0.0582)$ & $(0.136)$ & $(0.146)$ & $(0.292)$ & $(0.282)$ & $(0.233)$ \\
\hline \multirow[t]{2}{*}{ Cost } & $-0.0144 *$ & & & & & \\
\hline & $(0.00077)$ & & & & & \\
\hline \multirow[t]{2}{*}{ ASC } & $-1.02 *$ & & & & & \\
\hline & $(0.120)$ & & & & & \\
\hline \multirow{2}{*}{$\mu_{n}$} & $-2.23 *$ & & & & & \\
\hline & $(0.110)$ & & & & & \\
\hline$\#$ sig. attributes ${ }^{\mathrm{a}}$ & 11 & 1 & 7 & 4 & 3 & 4 \\
\hline Final LL & -5194.2 & & & & & \\
\hline Adj. rho-squared & $\begin{array}{l}0.174 \\
5808\end{array}$ & & & & & \\
\hline
\end{tabular}

Note: Figures in parenthesis report standard errors. * indicates statistical significance at or above the $95 \%$ level.

${ }^{a}$ In this row the number of significant estimates for the qualitative attribute level parameters is reported.

\subsubsection{Extending the SNA approach by incorporating reasons for non-attendance}

In model 4 we incorporate the five different behavioural reasons for attribute nonattendance obtained from supplementary questions as displayed in figure 1 . We identify five different scale function parameters depending on the reasons for not attending to the specific attribute as described in equation (4). We estimate and test each of these scale parameters relative to the scale parameter associated with the considered attributes $\left(\lambda_{\text {considered }}\right)$, which is fixed to one. Thus, the scale function essentially weights the ignored attributes' contribution to the likelihood function relative to the considered attributes. The overall results of model 4 show that three out of five scale parameters associated with the five different reasons for ignoring attributes are significantly lower than the reference level, $\lambda_{\text {considered }}$. This implies that the impact on the likelihood function of these observations is lower than for the observations for considered attributes. In more detail, the results of model 4 show that the scale parameter attached to the reason 'It is not important to me' ( $\lambda_{\text {ignored_reason } 1}$ ) with a value of 0.007 differs from one at a very high level of statistical significance. Hence, the attributes which are 
ignored because they are not important to respondents have not affected their choices in any way $^{7}$, implying that they actually contribute with the value zero (or very close to zero) to the likelihood function. This is in accordance with the standard SNA approach of assigning a zero weight to ignored attributes. However, this result also shows that when respondents claim to have ignored an attribute they might be speaking the truth and acting in line with the passive bounded rationality assumptions. Intuitively this would suggest that these observations should be allowed to contribute to the likelihood function rather than forcefully being restricted to zero. These findings are slightly in contrast to the findings of Carlsson et al. (2010) and Campbell and Lorimer (2009), but as we shall see this only applies to this particular reason.

Model 4 also shows that when respondents state that they ignored an attribute because 'It made it easier to choose between alternatives', the corresponding scale parameter ( $\left.\lambda_{\text {ignored_reason } 2}\right)$ is significantly different from one with a value of 0.202 . In line with Campbell and Lorimer (2009) and Carlsson et al. (2010), this result suggests that even though the attribute is clearly not fully considered in the choice task, it is not completely ignored since it contributes to the likelihood function. Moreover, the same results are obtained when attributes are ignored because their 'levels were unrealistically high or low', though the corresponding scale parameter $\left(\lambda_{\text {ignored }}\right.$ reason 3$)$ is not significantly different from one. Further analysis of the results from model 4 indicates that when the reason 'I don't think that this attribute should be weighed against the others' is taken into account ( $\left.\lambda_{\text {ignored reason } 4}\right)$ the results imply that respondents do not completely ignore those attributes either. Finally, a few individuals state that they are unsure about why they did not take certain attributes into account during the choice task. When this reason is incorporated into the analytical model ( $\left.\lambda_{\text {ignored_reasons }}\right)$ the results show that also with respect to this reason, respondents have not completely ignored the attribute.

All the above estimated scale parameters suggest that when ignoring attributes for one reason or another, the error variance or 'noise' for the observations associated with the ignored attributes becomes larger than for the considered attributes. Thus, the impact on choices from attributes ignored for the reason resulting in the largest error variance ('It is not important to me') can be seen as almost completely random for that specific attribute.

In model 5 we separate out the effect of the five reasons for ignoring attributes on attribute level by estimating a model where separate parameters are specified depending on the reasons for ignoring an attribute. In this case, each attribute level is interacted with the reasons for ignoring in order to catch the impacts of the corresponding reason on individual preferences. The results from this model are partly in line with model 4, in which all attribute levels with the exception of Quality2 are statistically insignificant when they are interacted with 'It is not important to me' (Ignored_reason1). Hence, these attributes do not really affect the choices of decision makers, and choices between these attribute levels are almost random. Furthermore, as discussed in section 5.2, this reason for ignoring an attribute is in line with the standard assumptions under the passive bounded rationality model. Thus, there is no reason to treat these observations any differently than attributes which have not been ignored. Except for a single attribute, it is evident that respondents have actually ignored the attributes they state. Restricting these attributes to zero has a very small impact on estimated parameters and removes some of the 'noise' from the model, giving rise to a structurally better fit. However, according to the passive bounded rationality assumptions, the statement 'It is not important to me' manifests genuine preferences, and thus, the intuition is that treating these attributes as if they are not ignored in the choice set would be more appropriate.

\footnotetext{
${ }^{7}$ Considering the inverse relationship between the scale parameter and the unexplained error variance (Swait and Louviere 1993), this implies that if the respondents were to make their choices solely based on this ignored attribute, their choices would be completely random.
} 
When examining the estimates for the second reason 'It made it easier to choose between alternatives' (Ignored_reason2) in model 5, the results show that seven of the estimated parameters are statistically significant (Number2, Catch2, Catch1, Nature2, Quality2, Quality1, and Size2). This result suggests that respondents stating this as a reason for ignoring attributes actually take some of the attributes into account to some extent, and for the remaining attributes/levels adopt some kind of decision strategy where by 'ignoring' the attribute, they reduce the information processing costs, thus making it easier to decide amongst the different alternatives. As discussed in section 5.2, this could be in support of the rationally adaptive behaviour model. Considering that Quality is the least ignored attribute and at the same time the highest ranked attribute in the preference ordering, it is not surprising that 'It made it easier to choose between alternatives' is the most often stated reason for ignoring the Quality attribute. This is evidence in support of Carlsson et al.'s (2010) suggestion that some respondents might state that they ignore attributes when in fact they just put less weight on them. While Quality is obviously a complex and thus information processing costly attribute which might cause respondents to ignore it, the fact that it is the highest valued attribute could pull in the opposite direction in terms of maximising benefits under the rationally adaptive behavioural model. Thus, some respondents might take it into account, but not to the extent that they would have liked due to the information processing costs, and as a consequence they state to have ignored it. In this case, the parameter estimates for Ignored_reason 2 in model 5 would actually reflect genuine preferences under rationally adaptive behaviour. The implied weighting in the scale function in model 4 would represent one way to make the rationally adaptive model operational in practice.

When respondents ignore attributes because 'the levels were unrealistically high or low' (Ignored_reason3) model 5 shows that four attribute levels (Catch1, Quality2, Quality1, and Size2) are statistically significant. Likewise, three attributes (Number2, Nature2, and Size1) are significantly different from zero when the reason 'I don't think that this attribute should be weighed against the others' is stated as the reason for ignoring attributes (Ignored_reason4). This suggests that for these attributes which are claimed to have been ignored, respondents to some extent have not completely ignored them. However, as a result of the underlying behavioural decision strategy of either protesting or using the satisficing, the lexicographic or the elimination by aspects heuristic as argued in section 5.2, we have no reason to expect that these estimates reflect genuine preferences. Hence, the standard approach of assigning a zero-contribution to the likelihood function would seem appropriate.

Finally, when respondents state 'Do not know' as a reason for why they exhibit attribute non-attendance behaviour (Ignored_reason5), four attributes (Number1, Catch2, Distance1, and Size1) are statistically significant. As argued in section 5.2 we cannot identify the underlying behavioural decision strategy supporting this reason, and due to this it is not possible to conjecture whether or not these estimates represent preferences in accordance with the passive bounded rationality model, the rationally adaptive behaviour model, or neither.

\subsubsection{Willingness to pay}

As previously mentioned, we have no information about price attribute nonattendance in the current dataset. Even though this does not invalidate our findings concerning the quality attribute preference parameters, we have chosen not to present detailed results concerning impacts of non-attendance and underlying reasons on WTP estimates. We believe that information concerning the actual level of attendance to the price attribute would be necessary in order to give an in-depth account of this. While we leave a thorough treatment of the potential impacts of underlying decision strategies on WTP and policy implications to future research which can be targeted more directly at this issue, we have of 
course calculated WTP estimates for all models ${ }^{8}$, and a few comments are in place. Interestingly, we found no significant differences in attribute WTP estimates across the five models. We do however not wish to draw the general conclusion that attribute non-attendance does not affect WTP, since this is conditional on the assumption that respondents are paying full attendance to the price attribute. Previous studies have shown this to be an unrealistic assumption (e.g. Campbell et al. 2008; Carlsson et al. 2010). Nevertheless, our results would suggest that if non-attendance is found to affect WTP, it is not driven by non-attendance to quality attributes but rather to the price attribute. Turning to the WTP estimates obtained under the five different reasons for non-attendance as specified in model 5, we also find no differences between these. Moreover, none of these are significantly different from the WTP estimates for the 'Ignored' estimates in model 3. This would suggest that nothing is gained in terms of WTP when going from the aggregate representation of non-attendance in model 3 to the more detailed specification of non-attendance according to underlying reasons in model 5. Again however, this is conditional on respondents paying full attention to the price attribute, so instead of drawing any conclusions in this regard we encourage researchers to address this important issue in future research.

\section{Conclusion}

The aim of this paper is to explore the implications of the behavioural reasons underlying respondents' attribute non-attendance in CE as well as testing analytical approaches to take these reasons explicitly into account. We use a novel follow-up question to identify potential reasons and attribute processing strategies underlying statements of attribute non-attendance.

Comparing to a benchmark model assuming continuous preferences, we present results from the standard way of dealing with stated attribute non-attendance as well as the approach suggested by Campbell and Lorimer (2009). While the former restricts ignored attributes to zero, the latter allows ignored attributes to be freely estimable. Our results confirm the findings of Campbell and Lorimer (2009) as well as Carlsson et al. (2010) that the standard approach of fixing ignored attributes to zero may not be appropriate. We suggest a novel extension of the current SNA approach where we utilise the stated reasons for ignoring attributes identified in the follow-up question mentioned above. Based on two different econometric models we show that the behavioural reasons underlying statements of attribute non-attendance have important bearings on the appropriateness of the standard SNA approach. For respondents who state that they ignore attributes simply because the attributes do not matter to them, our models confirm that they have actually ignored the attributes. Hence, the standard SNA approach of assigning a fixed zero contribution to the likelihood function from these attributes might produce biased results, since these genuine zero preferences are excluded from the mean parameter estimate. Also intuitively, it would be more appealing to treat these observations as non-ignored attributes since they reflect genuine preferences in accordance with the passive bounded rationality assumptions. Therefore, we suggest recoding of these non-attendance statements from ignored to non-ignored. For respondents who state that they ignore attributes to make the choice tasks easier, we find that the standard SNA approach is inappropriate since attributes claimed to have been ignored are actually not completely ignored according to the econometric models. We argue along the rationally adaptive behavioural model that the preference parameter estimates picked up by the analytical model reflect genuine preferences, though they have to be given less weight in the econometric models than preferences elicited from non-ignored attributes. We propose a scaling approach to estimate and incorporate such weighting procedures. The findings from

\footnotetext{
${ }^{8}$ Available from the authors upon request.
} 
these first two reasons for non-attendance suggest that we need to improve the SNA approach to better distinguish between non-attendance and low preferences. Some respondents stated that they ignored attributes because they found the levels unrealistic, or they found it inappropriate to trade-off these attributes against others, and a few respondents were not able to explain why they ignored attributes. Even though some of the preference parameter estimates are found to be significant under these three reasons, we argue that the behaviour underlying these reasons imply that estimates should not be given a preference interpretation since it is not in accordance with neither the passive bounded rationality model nor the adaptive rational model. Hence, the standard SNA approach would seem appropriate when these reasons underlie stated non-attendance.

In response to Carlsson et al.'s (2010) call for attention to the reasons underlying stated non-attendance, our survey represents a first attempt to explore this area. Both Carlsson et al. (2010) and Campbell and Lorimer (2009) have questioned the standard SNA approach of fixing the likelihood contribution of ignored attributes to zero. Our results strongly underline the importance of focusing on underlying behaviour as we are able to show that it is not a matter of either using the standard approach or not, but rather a matter of using the standard approach to some extent conditional on the specific reasons for nonattendance. In particular, our results suggest that using the standard approach in combination with weighting procedures and recoding of non-attendance statements conditional on stated reasons would be an appropriate solution. As this paper only represents a first effort, much more research is of course needed in order to thoroughly investigate the impacts and implications of the behavioural decision processes underlying attribute non-attendance. Following Scarpa et al. (2010) and Puckett and Hensher (2009), one potentially important area for future research would be to investigate the impact of behavioural reasons underlying attribute non-attendance at the choice task level rather than at the choice sequence level presented here. 


\section{References}

Bateman, I. J.; Burgess, D.; Hutchinson, W. G. and Matthews, D., (2008). Learning design contingent valuation (LDCV): NOAA guidelines, preference learning and coherent arbitrariness. Journal of Environmental Economics and Management, vol. 55(2) pp.127-141

Bierlaire, M. (2003). BIOGEME: A free package for the estimation of discrete choice models , Proceedings of the 3rd Swiss Transportation Research Conference, Ascona, Switzerland.

Campbell, D., (2008). Identification and analysis of discontinuous preferences in discrete choice experiments. European Association of Environmental and Resource Economics Annual Conference, Goteborg, Sweden.

Campbell, D., Hutchinson, W., H., and Scarpa, R., (2008). Incorporating discontinuous preferences in to the analysis of discrete choice experiments. Environmental and Resource Economics 41 pp. 401 - 417

Campbell, D, and Lorimer, VS, (2009). Accommodating attribute processing strategies in stated choice analysis: do respondents do what they say they do? European association of environmental and resource economists annual conference, Amsterdam.

Campbell, D, Lorimer, VS, Aravena, C, and Hutchinson, G (2010). Attribute processing in environmental choice analysis: implications for willingness to pay. Agricultural Economics Society Annual Conference, Edinburgh, 29-31 March 2010.

Carlsson, F., Kataria, M., and Lampi, E., (2010). Dealing with ignored attributes in choice experiments on valuation of Sweden's environmental quality objectives. Environmental and Resource Economics 47: 65-89.

DeShazo, J., R., and Fermo, G., (2002). Designing choice sets for stated preference methods: the effect of complexity on choice consistency. Journal of Environmental Economics and Management 44: pp. 123 - 143.

DeShazo, J., R., and Fermo, G., (2004). Implications of rationally-adaptive pre-choice behaviour for the design and estimation of choice models. University of California, Los Angeles.

Gowdy, J., M., and Mayumi, K., (2001). Reformulating the foundations of consumer choice theory and environmental valuation. Ecological Economics 39: 223-237.

Greene WH and Hensher DA (2007). Heteroscedastic control for random coefficients and error components in mixed logit. Transport Research part E 43(5):610-623

Hensher, D., A., Rose, J., and Green, W., H., (2005a). The Implication on willingness to pay of respondents ignoring specific attributes. Transportation 32: pp. $203-222$.

Hensher DA, Rose JM, Greene WH (2005b). Applied choice analysis: a primer. Cambridge University Press, Cambridge

Hensher DA and Greene WH (2003). The mixed logit model: the state of practice. Transportation 30(2):133-176

Hensher, D., A., (2006). How do respondents process stated choice experiments? Attribute consideration under varying information load. Journal of Applied Economics 21: pp. 861-878. 
Hensher, D.A. (2010). Attribute Processing, Heuristics and Preference Construction in Choice Analysis. (Invitational Keynote Paper for Choice Modelling Conference, Leeds UK. March 30-April 1 2009, linked ARC DP 0770618; 07-09). in Hess, S. and Daly, A. (eds.) State-of Art and State-of Practice in Choice Modelling, Emerald Press, UK., 35-70.

Hensher, D., A., Rose, J., and Bertoia, T., (2007). The implication on willingness to pay of a stochastic treatment of attribute processing in stated choice studies. Transportation research part $E$ 43: pp. $73-89$.

Hensher, D., A., and Greene, W., H., (2010). Non-attendance and dual processing of common-metric attributes in choice analysis: a latent class specification. Empirical economics 39(2) 413-426.

Hess, S. \& Rose, J.M. (2009). Allowing for intra-respondent variations in coefficients estimated on stated preference data, Transportation Research B, 43(6), pp. 708-719.

Hole, A., R., (2010). A discrete choice model with endogenous attribute attendance. Sheffield economic research paper series, Sheffield, UK.

Hoyos, D., Mariel, P., and Meyerhoff, J., (2010). Comparing the performance of different approaches to deal with attribute non-attendance in discrete choice experiments: a simulation experiment. BILTOKI 201001, Universidad del País Vasco Departamento de Economía Aplicada III (Econometría y Estadística).

Jorgensen, B. S., G. J. Syme, B. J. Bishop, \& B. E. Nancarrow (1999). Protest Responses in Contingent Valuation. Environmental and Resource Economics. Vol. 14(1) pp. 131150 .

Kaye-Blake, W., H., Abell, W., L., and Zellman, E., (2009). Respondents' ignoring of attribute information in a choice modelling survey. Agricultural and Resource Economics, pp. $547-564$.

Lancsar, E., and Louviere, J., J., (2006). Deleting 'irrational' responses from discrete choice experiment: a case of investigating or imposing preferences? Health Economics 15: 797-811

Lockwood, M., (1996). Non-compensatory preference structure in non-market valuation of natural area policy. Australian journal of Agricultural economics 40: pp. 85-101.

Louviere, J., J., Hensher, D., A., and Swait, J., D., (2000). Stated choice methods: analysis and application. Cambridge university press, Cambridge.

Lundhede TH, Olsen SB, Jacobsen JB, Thorsen BJ (2009). Handling respondent uncertainty in choice experiments: Evaluating recoding approaches against explicit modelling of uncertainty. Journal of Choice Modelling 2(2):118-147

Meyerhoff J, Liebe U (2006). Protest beliefs in contingent valuation: explaining their motivation. Ecological Economic 57(4):583-594

Meyerhoff, J., Liebe, U. (2009). Discontinuous preferences in choice experiments: Evidence at the choice task level. Paper presented at the 2009 EAERE Conference, Amsterdam.

Mitchell, R. C. \& R. T. Carson (1989): Using Surveys to Value Public Goods: The Contingent Valuation Method. Resources for the Future, Washington D.C., USA

Morrison MD, Blamey RK, Bennett JW (2000). Minimising payment vehicle bias in contingent valuation studies. Environmental and Resource Economics 16(4):407-422 
Payne, J., W., Bettman, J., R, Coupey, E., Johnson, E. J, (1992). A constructive process view of decision making: multiple strategies in judgment and choices. Acta Psycologica 80, 107-141.

Puckett, S. M., Hensher, D. A., (2008). The role of attribute processing strategies in estimating the preferences of road freight stakeholders. Transportation Research part E 44, 379-395

Puckett, S., M., and Hensher, D., A., (2009). Revealing the extent of preference heterogeneity in choice analysis: an empirical assessment. Transportation Research part A 43: pp. 117-126.

Rosenberger, R., S., Peterson, G., L., Clarke, A., and Brown, T., C., (2003). Measuring dispositions for lexicographic preferences of environmental goods: integrating economics, psychology and ethics. Ecological economics 44: pp. 63-76.

Ryan, M., Watson, V., and Entwistle, V., (2009). Rationalizing the irrational: a think aloud study of discrete choice experiment responses. Health economics 18: pp. 321-326.

Sælensminde, K. (2001). Inconsistent choices in stated choice data: use of the logit scaling approach to handle resulting variance increases. Transportation 28: 269-296.

Scarpa, R., Ruto, E. S. K., Kristjanson, P., Radeny, M., Drucher, A. G. and Rege, J. E. O., (2003). Valuing indigenous cattle breeds in Kenya: an empirical comparison of stated and revealed preference value estimates. Ecological Economics, 45, 409-426.

Scarpa, R., Gilbride, T., J., Campbell, D., and Hensher, D., H., (2009). Modelling attribute non-attendance in choice experiment for rural landscape valuation. European review of Agricultural Economics 36: pp. 151-174.

Scarpa R, Ferrini S, Willis K (2005). Performance of error component models for status-quo effects in choice experiments. In: Scarpa R, Alberini A (eds) Applications of simulation methods in environmental and resource economics. The economics of nonmarket goods and resources, vol 6. Springer, Dordrecht, pp 247-273

Scarpa, R., Willis, K. and Acutt, M., (2007). Valuing externalities from water supply: status quo, choice complexity, and individual random effects in panel kernel logit analysis of choice experiments. Journal of Environmental Planning and Management, 50, 449-466.

Scarpa R, Thiene M, Marangon F (2008) Using flexible taste distributions to value collective reputation for environmentally friendly production methods. Canadian Journal of Agricultural Economics 56(2):145-162

Scarpa, R., Thiene, M., \& Hensher, D. A. (2010). Monitoring Choice Task Attribute Attendance in Nonmarket Valuation of Multiple Park Management Services: Does It Matter? Land Economics 86(4), 817-839.

Swait, J., and Louviere, J., (1993). The role of the scale parameter in the estimation and comparison of multinomial logit models. Journal of Marketing Research 30: pp. 305314.

Train, K., E., (2003). Discrete choice methods with simulation. Press Syndicate of the University of Cambridge. 